\title{
A Kind of Color Two-dimensional QR Barcode Design
}

\author{
LiDong $^{1, a^{*}, \text { Xie Hongan }}{ }^{2, b}$ \\ ${ }^{1}$ Information engineering department, Engineering College of CAPF, \\ Xi'an, Shanxi,China \\ ${ }^{2}$ Electronics department, Engineering College of CAPF, \\ Xi'an, Shanxi,China \\ a email: 563783912@qq.com \\ bemail:15529332695@163.com
}

Keywords: black and white two-dimensional barcode; color two-dimensional QR bar code; coding; coding modes

Abstract. Accommodate the problem of the amount of information for the general black and white two-dimensional bar code is relatively small and the Chinese character coding efficiency is relatively low, we propose a color two-dimensional QR barcode.This scheme focuses on the color two-dimensional QR barcode encoding process of character coding mode for a detailed explanation. Through the character encoding mode performance analysis, combined with the front of the bar code capacity, analysis results show that the scheme of the color two-dimensional bar code increased dimensional bar code information and enhanced Chinese character coding efficiency.

\section{Introduction}

With the rapid development of global information and communications technology,various applications for mobile termina continue to emerge, the phone undoubtedly become one of the most important terminals which the development of mobile commerce depends on, and the development of the two dimensional barcode will play an important role in the development of mobile commerce ${ }^{[1-3]}$.

Currently with the rapid development of bar code technology, people put forward new challenges about the amount of information of the bar code ${ }^{[4]}$,theoretically increasing density barcode or increasing code size exists some problems ${ }^{[5]}$. This paper introduces a color QR code which does not change the size and density of the bar code, at the same time increases the amount of barcode information, while the two-dimensional code becomes more beautiful.

Firstly, considering the QR code in reading speed, the amount of information and other aspects are better than other commonly used two-dimensional code; In addition, due to the QR code of Chinese characters information has a unique function of optimization, in line with the Chinese characters habits. so the research on color QR two-dimension bar code technology has a far-reaching significance, which has wide application prospect in our country.

\section{Design Concept of Colored QR Barcode}

It is important to select the color of the module for color QR code. In the selection of the QR code module color should follow the.principle of the the strongest contrast color and most of the difference value.

Due to the color of two dimensional barcode identification involves the problem of the color image segmentation $^{[6]}$, the most commonly used color image segmentation method is based on RGB color space model, this method is simple and the effect is very good ${ }^{[7]}$.The closeness of the colors between coding region and the background of logo to some extent determines the appearance of the QR 
code.In order to ensure that the design of the QR code to better highlight the logo,color selection is based on the logo background color, to the color coding used as close as logo background color.

When selecting the color used in the coding region, firstly, extracting the background color of the Logo, and then obtaining the average value of the background color RGB. Assuming the pixels at the Logo graphics background region are $m \times n$, the $R G B$ value of each pixel is $\left(r_{i}, g_{i}, b_{i}\right), i=1,2 \cdots, m n$,By averaging the RGB values of all background pixels obtained the average RGB values for $(\overline{\mathrm{r}}, \bar{g}, \bar{b})$, then:

$$
\bar{r}=\frac{r_{1}+r_{2}+\cdots+r_{m n}}{m n}, \bar{g}=\frac{g_{1}+g_{2}+\cdots+g_{m n}}{m n}, \quad \bar{b}=\frac{b_{1}+b_{2}+\cdots+b_{m n}}{m n}
$$

After getting the average value $(\overline{\mathrm{r}}, \bar{g}, \bar{b})$, select the color that is close to it. we choose four kinds of coding as examples:

Because of the selection of four colors, so we use quaternary encoding. The four colors are $(a, b, b) 、(b, a, b) 、(b, b, a) 、(a, a, b)$. In order to better identify,the color selection of the RGB must be able to meet $(\mathrm{a}-\mathrm{b}){ }^{*} \mathrm{n}=\mathrm{a}$ (and $\mathrm{N}$ is positive integer). Now we attempt to a $\log$ as an example.the logo of the background color is white, so the color selection is close to white. we can make $\mathrm{a}=255, \mathrm{n}=5, \mathrm{~b}=200$, so we can pick and choose the colors: $(200,250,200)$ 、 $(250,250,200)$ 、 $(250,200,200)$ 、 (200,200,250), and the color value we can choose are many.

Next we make the following rules: with quaternary numbers $0,1,2,3$, respectively (250,200,200), (250,250,200), (200,250,200) and (200,200,250) four colors, as shown in Table 1.

\begin{tabular}{|c|c|c|c|c|}
\hline $\begin{array}{c}\text { The RGB of } \\
\text { selected color }\end{array}$ & $(250,200,200)$ & $(250,250,200)$ & $(200,250,200)$ & $(200,200,250)$ \\
\hline $\begin{array}{c}\text { Corlor } \\
\text { display }\end{array}$ & & & & \\
\hline Quatenary & 0 & 1 & 2 & 3 \\
\hline
\end{tabular}

Table 1

\section{Coding of color QR code}

Because the code of the Quaternary,we need to turn the information into value firstly, and then converted into quaternary data. Finally, add quaternary mode indicator and character indicator in front of the data after conversion.

We take the digital coding model as an example: Assuming that $\mathrm{N}$ represents the number of digits to be encoded, $r$ is the number of digits remaining in the back of the corresponding packet.Due 
to $9 \longrightarrow 21,99 \longrightarrow 1203,999 \longrightarrow 33213,9999 \longrightarrow 2130033$.We can get that two decimal numbers can represented by four quaternary number; three decimal numbers can represented by five quaternary numbers, up to four decimal numbers represented by seven quaternary numbers. We can get that after the analysis of the total length of the bit stream of the input data packet can be analyzed:

Two bit one group: the total length of the bit stream $S=\frac{N}{2} \times 4+R\left\{\begin{array}{l}\mathrm{r}=0, R=0 \\ \mathrm{r}=1, R=2\end{array}\right.$

Three bit one group: the total length of the bit stream

$$
S=\frac{N}{3} \times 5+R\left\{\begin{array}{l}
\mathrm{r}=0, R=0 \\
\mathrm{r}=1, R=2 \\
\mathrm{r}=2, R=4
\end{array}\right.
$$

Four bit one group: the total length of the bit stream

$$
S=\frac{N}{4} \times 7+R\left\{\begin{array}{l}
\mathrm{r}=0, R=0 \\
\mathrm{r}=1, R=2 \\
\mathrm{r}=2, R=4 \\
\mathrm{r}=3, R=5
\end{array}\right.
$$

\section{Analysis of Data Capacity and Aesthetics of Colored QR Code}

In this artical, the coding used $n(n>2$, and as an integer) colors to replace the black and white. Assuming that the two-dimensional code is composed of K pixels, then the capacity by $2^{k}$ bar code is $n^{k}$, the amount of information on the color QR code into a black QR code $\left(\frac{n}{2}\right)^{k}$ times. So as long as $n>2$,it is able to enhance the capacity of two-dimensional code.In this paper,the logo will not cover the coding region, can greatly improve the error correction capability.we use the quaternary encoding as example, obtained compression ratio by analyzing and comparing the color QR Code and the black and white QR Code without the addition of logo graphic case, it can be seen that using quaternary encoding color QR code can promote compression ratio a lot. as shown in Table 2.

\begin{tabular}{|c|c|c|c|c|}
\hline Coding mode & $\begin{array}{c}\text { Digital } \\
\text { coding } \\
\text { mode }\end{array}$ & $\begin{array}{c}\text { Alphanumeric } \\
\text { code model }\end{array}$ & $\begin{array}{l}\text { 8-bitbytes } \\
\text { /JIS8 } \\
\text { character } \\
\text { encoding } \\
\text { mode }\end{array}$ & $\begin{array}{c}\text { Chinese } \\
\text { character } \\
\text { coding } \\
\text { mode }\end{array}$ \\
\hline $\begin{array}{c}\text { Black and white QR } \\
\text { code }\end{array}$ & $1: 3.3$ & $1: 5.5$ & $1: 8$ & $1: 13$ \\
\hline Corlor QR code & $1: 1.7$ & $1: 3$ & $1: 4$ & $1: 6$ \\
\hline
\end{tabular}

Table 2

From Figure 1 can be seen that as a result of relatively light color, coupled with relatively larger logo area, greatly enhancing the appearance of the two-dimensional code. If the information we add is 
small enough, can further enhance the logo region.

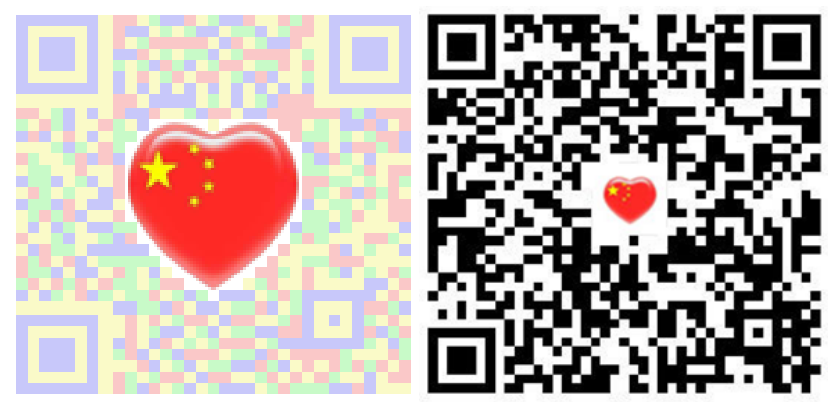

Figure 1

\section{Summary}

white two-dimensional code is still of some defects.In view of the small data capacity and the poor aesthetics of the black-and-white 2D code,this paper puts forward a method to design the colored QR code, and gives a specific method for generating and encoding. By the way of reducing color saturation, to enhance the density of information coding and makes the logo more prominent; Since modules are arranged around the logo, it will not cause the loss of modules, enhancing the error correcting capability of two-dimensional code.

This design of QR codes fully consider the market demand applications, which has great practical value.

\section{References}

[1]QR Code:Technology and Application[M],compiled by Article Numbering Center of China, Beijing: China Standards Press,2002.

[2]HAN Guo Zhi.Error correction coding theory and MATLAB realization[N].Science and Technology for China's Mass Media. 2007,No.4:17-22.

[3]GB17172-1997.Portable Data File[S].BeiJing:China Standard Press.1997.

[4]HUANG Nai Ying,ZHAN Yi Ju.Design of color three-dimensional PDF417 barcode[J].Automation and information,2011,3:9-15.

[5]LIU Ning Zhong.Research on recognition technology and encoding theory of hign-dimensional bar code[D].Nanjing University of Science and Technology,2003.

[6]Ottaviani E,Pavan A,et al.A common image processing framework

for 2D barcode reading.Image Processing and its Applications[C].Conference Publication, IEEE,1999(465): 652-655.

[7]Hwang Suk-Seung,John J Shynk.GPS AOA estimation based on a modified despreader[C].Conference Record of the Asilomar Conference on Signals,Systems and Computers,2009:1140-1143. 